\title{
Some integrability estimates for solutions of the fractional $p$-Laplace equation
}

\section{Shaoguang Shi}

Department of Mathematics, Linyi University, Linyi 276005, China.

\author{
Communicated by $\mathrm{Y}$. Hu
}

\begin{abstract}
For $(\alpha, p) \in(0,1) \times(1, \infty)$, this note focuses on some integrability estimates for solutions of the following Dirichlet problem

$$
\begin{cases}\mathrm{L}_{\alpha, p} \mathrm{u}(\mathrm{x})=\mathrm{g}(\mathrm{x}) & \text { as } x \in \Omega, \\ \mathfrak{u}(x)=0 & \text { as } x \in \mathbb{R}^{\mathfrak{n}} \backslash \Omega,\end{cases}
$$
\end{abstract}

where $\mathrm{L}_{\alpha, p}$ is the fractional p-Laplace operator. (C)2017 All rights reserved.

Keywords: Fractional p-Laplace equation, Dirichlet problem, solution.

2010 MSC: 35R11, 31C05, 42B35.

\section{Introduction}

Unless stated otherwise, we will always assume that $(\alpha, p) \in(0,1) \times(1, \infty)$ and $\Omega$ is a bounded Lipschitz domain. This paper is devoted to a further study of the integrability estimates for weak solutions of the following Dirichlet problem

$$
\begin{cases}\mathrm{L}_{\alpha, p} \mathrm{u}(x)=g(x) & \text { as } x \in \Omega, \\ \mathfrak{u}(x)=0 & \text { as } x \in \mathbb{R}^{\mathfrak{n}} \backslash \Omega .\end{cases}
$$

Here $L_{\alpha, p}$ is the so-called fractional $p$-Laplace operator

$$
\mathrm{L}_{\alpha, p} \mathrm{u}(\mathrm{x})=\text { p.v. } \int_{\mathbb{R}^{\mathrm{n}}} \frac{|\mathrm{u}(\mathrm{y})-\mathfrak{u}(\mathrm{x})|^{\mathrm{p}-2}(\mathrm{u}(\mathrm{y})-\mathrm{u}(\mathrm{x}))}{|y-x|^{\mathfrak{n}+\alpha p}} \mathrm{dy} .
$$

When $p=2, L_{\alpha, 2}$ has already been known as the classical fractional Laplace operator, which has initially been studied $([11,18])$. It is a generator of a strongly continuous contractive semigroup on $\mathrm{L}^{2}\left(\mathbb{R}^{\mathrm{n}}\right)$ that can be extended to contraction semigroup on $\mathrm{L}^{\mathrm{p}}\left(\mathbb{R}^{\mathrm{n}}\right)$ for $p \in[1, \infty]([3,8])$. The Dirichlet boundary problem of $\mathrm{L}_{\alpha, 2}$ has been intensively investigated and many fundamental results have been proved, we refer the reader to $[2,4,8,12,14]$ and the references therein for a fuller treatment of this topic. As a nonlinear generalization of $L_{\alpha, 2}, L_{\alpha, p}$ has been extensively explored in recent years $([1,5,10])$.

Email address: shishaoguang@mail.bnu.edu.cn (Shaoguang Shi)

doi:10.22436/jnsa.010.10.38 
When $g=-\lambda|u|^{p-2} u$ with $\lambda>0$, the equation

$$
\mathrm{L}_{\alpha, p} u(x)=-\lambda|u(x)|^{p-2} u(x),
$$

which also called the nonlocal Euler-Lagrange equation was fully discussed in [13] for large values of $p$ and the limit equation as $p \rightarrow \infty$ was derived. Equation (1.2) was closely related to the nonlocal eigenvalue problem and its viscosity solutions have many interesting properties. If $g=-|u(x)|^{p-2} u(x)$, a local version of (1.1), i.e.,

$$
\begin{cases}\mathrm{L}_{\alpha, \mathrm{p}} \mathrm{u}(\mathrm{x})=-|\mathrm{u}(\mathrm{x})|^{\mathrm{p}-2} \mathrm{u}(\mathrm{x}) & \text { as } x \in \Omega, \\ \mathfrak{u}(\mathrm{x})=1 & \text { as } x \in \mathrm{K}, \text { compact } \mathrm{K} \subset \Omega, \\ \mathrm{u}(\mathrm{x})=0 & \text { as } x \in \mathbb{R}^{\mathfrak{n}} \backslash \Omega,\end{cases}
$$

was studied in $[15,16]$. It was proved that the weak solution of $(1.3)$, which was nothing but the viscosity solution, was the capacitary potential of the relative fractional Sobolev capacity.

In [1], Barrios et al. studied the summability of the finite energy solutions to (1.1) in terms of the summability of $g$ for $\alpha p<n$ by adapting the ideas used in [12] for $p=2$. In this paper, highly inspired by their methods and some known estimates for bounded Lipschitz domain, we obtain the following results for $\alpha p \geqslant n$.

Theorem 1.1. Let $\mathrm{g} \in \mathrm{L}^{\mathrm{s}}(\Omega), \mathrm{p} \leqslant \mathrm{q}<\infty$ with $\alpha \mathrm{p}=\mathrm{n}$ and $\Omega$ be $a \mathrm{~W}^{\alpha, \mathrm{p}}$-extension domain. Then the solution of (1.1), denoted by $\mathrm{u}$, satisfies the following boundedness:

(a) for $s>\frac{\alpha q}{\alpha q-n}$, there exists a constant $C_{1}:=C_{1}\left(n, \alpha, q, \Omega,\|g\|_{L^{s}(\Omega)}\right)$ such that

$$
\|u\|_{L^{\infty}(\Omega)} \leqslant C_{1}
$$

(b) for $\mathrm{s}=\frac{\alpha \mathrm{q}}{\alpha q-\mathrm{n}}$, there exists a constant $\mathrm{C}_{2}:=\mathrm{C}_{2}\left(\mathrm{n}, \alpha, \mathrm{q}, \Omega,\|g\|_{\mathrm{L}^{\mathrm{s}}(\Omega)}\right)$ such that

$$
\int_{\Omega} \exp ^{\beta|u(x)|} \mathrm{d} x \leqslant C_{2} \text {, for some } \beta>0 \text {; }
$$

(c) for $\frac{\mathrm{q}}{\mathrm{q}-1} \leqslant \mathrm{~s}<\frac{\alpha \mathrm{q}}{\alpha \mathrm{q}-\mathrm{n}}$, there exists a constant $\mathrm{C}_{3}:=\mathrm{C}_{3}(\mathrm{n}, \alpha, \mathrm{s})$ such that

$$
\|u\|_{L^{*}(\Omega)} \leqslant C_{3}\|g\|_{L^{s}(\Omega)}^{\frac{\alpha}{n-\alpha}} \text { as } s^{*}=\frac{q s(n-\alpha)}{s(n-q \alpha)+q \alpha} .
$$

Theorem 1.2. Assume that $\mathrm{g} \in \mathrm{L}^{1}(\Omega), \alpha p>\mathrm{n}$ and $\mathrm{u}$ is a solution of (1.1). Then there exists a constant $\mathrm{C}:=\mathrm{C}\left(\mathrm{n}, \alpha, \mathrm{p}, \Omega,\|g\|_{\mathrm{L}^{1}(\Omega)}\right)$ such that

$$
\|u\|_{L^{\infty}(\Omega)} \leqslant C .
$$

We end this section with the outline of this paper. Section 2 presents some basic definitions and preliminaries. In Section 3, we give the proofs of Theorem 1.1 and Theorem 1.2.

\section{Preliminaries}

We recall that the inhomogeneous fractional Sobolev space $W^{\alpha, p}\left(\mathbb{R}^{n}\right)$ is defined as

$$
W^{\alpha, p}\left(\mathbb{R}^{n}\right):=\left\{f \in L^{p}\left(\mathbb{R}^{n}\right): \frac{|f(y)-f(x)|}{|y-x|^{\alpha+n / p}} \in L^{p}\left(\mathbb{R}^{n} \times \mathbb{R}^{n}\right)\right\}
$$

endowed with the norm

$$
\|f\|_{W^{\alpha, p}\left(\mathbb{R}^{n}\right)}:=\left(\int_{\mathbb{R}^{n}}|f(x)|^{p} d x+\int_{\mathbb{R}^{n}} \int_{\mathbb{R}^{n}} \frac{|f(y)-f(x)|^{p}}{|y-x|^{n+\alpha p}} d y d x\right)^{1 / p} .
$$


Also, $W^{\alpha, p}(\Omega)$ can be defined similarly with $\mathbb{R}^{n}$ replaced by $\Omega$. The homogeneous fractional Sobolev space $\dot{W}^{\alpha, p}\left(\mathbb{R}^{\mathfrak{n}}\right)$ can be defined by the semi-norm

$$
\|f\|_{\dot{W}^{\alpha, p}\left(\mathbb{R}^{n}\right)}:=\left(\int_{\mathbb{R}^{n}} \int_{\mathbb{R}^{n}} \frac{|f(y)-f(x)|^{p}}{|y-x|^{n+\alpha p}} d y d x\right)^{1 / p},
$$

which is the so-called Gagliardo semi-norm of $f$. Fractional Sobolev spaces have been a classical topic in functional and harmonic analysis all along, see e.g., the review paper [6] and the references therein. $\mathrm{C}(\Omega)$ is the space of all real-valued and continuous functions on $\Omega$. For each natural number $k$, i.e., $k \in \mathbb{N}, C^{k}(\Omega)$ denotes the space of all functions being $k$ times continuously differentiable, $C_{c}^{k}(\Omega)$ stands for the space of all functions in $C^{k}(\Omega)$ having compact support. $C_{\mathcal{c}}^{\infty}(\Omega)$ is the subspace of $C_{c}^{k}(\Omega)$ given by $C_{c}^{\infty}(\Omega):=\cap_{k} C_{c}^{k}(\Omega) . W_{0}^{\alpha, p}(\Omega)$ is the closure of $C_{c}^{\infty}(\Omega)$ in the norm $\|\cdot\|_{W^{\alpha, p}(\Omega)}$.

Theorem 1.1 is based on the assumption that $\Omega$ is an extension domain. We say that $\Omega$ is a $W^{\alpha, p_{-}}$ extension domain, if there is a positive constant $C:=C(n, p, \Omega, \alpha)$ such that for every function $f \in$ $W^{\alpha, p}(\Omega)$, there exists a function $\widetilde{f} \in W^{\alpha, p}\left(\mathbb{R}^{n}\right)$ with

$$
\widetilde{f}(x)=f(x) \text { as } x \in \Omega, \quad \text { and } \quad\|\widetilde{f}\|_{W^{\alpha, p}\left(\mathbb{R}^{\mathfrak{n}}\right)} \leqslant C\|f\|_{W^{\alpha, p}(\Omega)} .
$$

Fractional extension results are essential to improve some fractional embedding theorems and have been discussed by many people such as Nezza-Palatulli-Valdinoci [6], Shvartsman [17], Triebel [19] and Zhou [21]. It is well-known that the space $W^{\alpha, p}(\Omega)$ is continuously embedded in $L^{q}(\Omega)$ for any $q \in[p, \infty)$ when $\Omega$ is a $W^{\alpha, p}$-extension domain. That is:

Lemma 2.1. Let $\alpha \mathrm{p}=\mathrm{n}, \mathrm{q} \in[\mathrm{p}, \infty)$ and $\Omega$ be a $\mathrm{W}^{\alpha, \mathrm{p}}$-extension domain. Then there is a constant

$$
C:=C(\Omega, n, \alpha, p)>0,
$$

such that

$$
\|f\|_{L^{q}(\Omega)} \leqslant C\|f\|_{W^{\alpha, p}(\Omega)}, \quad \forall f \in W^{\alpha, p}(\Omega) .
$$

Moreover, if $\mathrm{f} \in \dot{\mathrm{W}}^{\alpha, \mathrm{p}}(\Omega) \cap \mathrm{C}_{\mathrm{c}}(\Omega)$, then

$$
\|f\|_{L^{q}(\Omega)} \leqslant C\|f\|_{\dot{W}^{\alpha, p}(\Omega)},
$$

where $C_{c}(\Omega)$ stands for all real-valued and continuous functions on $\Omega$ having compact support.

Proof. The first estimate is just [6, Theorem 6.10]. It only needs to prove the second one. If

$$
f \in \dot{W}^{\alpha, p}(\Omega) \cap C_{c}(\Omega),
$$

then supp $f:=K$ is a compact set, it follows from [9, Lemma 2.6] that $f \in L^{p}(K)$, i.e., there is a constant $\mathrm{C}:=\mathrm{C}(\mathrm{n}, \alpha, \mathrm{p}, \Omega)>0$ such that

$$
\|f\|_{L^{p}(K)} \leqslant C\|f\|_{\dot{W}^{\alpha, p}(\Omega)} .
$$

Consequently, one has

$$
\|f\|_{L^{q}(\Omega)} \leqslant C\|f\|_{W^{\alpha, p}(\Omega)}=C\left(\|f\|_{L^{p}(K)}+\|f\|_{W^{\alpha, p}(\Omega)}\right) \leqslant C\|f\|_{W^{\alpha, p}(\Omega)} .
$$

A function $u \in W^{\alpha, p}(\Omega)$ is a weak solution of (1.1) subject to the boundary condition $u(x)=0$ on $\mathbb{R}^{\mathfrak{n}} \backslash \Omega$ if

$$
\left\langle\mathrm{L}_{\alpha, \mathrm{p}} \mathrm{u}, \phi\right\rangle=\langle\mathrm{g}, \phi\rangle, \quad \forall \phi \in \mathrm{W}_{0}^{\alpha, \mathrm{p}}(\Omega),
$$

where

$$
\left\langle\mathrm{L}_{\alpha, p} \mathrm{u}, \phi\right\rangle:=\int_{\Omega} \int_{\Omega} \frac{|\mathrm{u}(\mathrm{y})-\mathrm{u}(\mathrm{x})|^{p-2}(\mathfrak{u}(\mathrm{y})-\mathrm{u}(\mathrm{x}))(\phi(\mathrm{y})-\phi(x))}{|y-x|^{n+\alpha p}} \mathrm{~d} \mathrm{~d} \mathrm{~d} x,
$$

and $\langle\mathrm{g}, \phi\rangle$ is given by the duality product. It follows from [7, Theorem 5.5] and [1, Theorem 2.6] that there exists a unique weak solution to (1.1). That is: 
Lemma 2.2. For any $\mathrm{f} \in \mathrm{W}^{-\alpha, \mathrm{p}^{\prime}}(\Omega)$, the dual space of $\mathrm{W}^{\alpha, \mathrm{p}}(\Omega)$, there is a unique function $\mathrm{u} \in \mathrm{W}^{\alpha, \mathrm{p}}(\Omega)$ such that

$$
\mathrm{L}_{\alpha, \mathrm{p}} \mathrm{u}=\mathrm{f} .
$$

The next lemma is a straightforward consequence of [1, Lemma 2.8, Proposition 2.10, Lemma 2.13], we collect here without their proofs.

Lemma 2.3. Let $u, v \in \dot{W}^{\alpha, p}(\Omega)$.

(a) If $\mathrm{F} \in \operatorname{Lip}(\mathbb{R})$ with $\mathrm{F}(0)=0$, then $\mathrm{F}(\mathrm{u}) \in \dot{\mathrm{W}}^{\alpha, \mathrm{p}}(\Omega)$. Furthermore, if $\mathrm{F}$ is a convex function and differentiable almost everywhere, one has

$$
\mathrm{L}_{\alpha, \mathrm{p}} \mathrm{F}(\mathrm{u}) \leqslant\left|\mathrm{F}^{\prime}(\mathrm{u})\right|^{\mathrm{p}-2} \mathrm{~F}^{\prime}(\mathrm{u}) \mathrm{L}_{\alpha, \mathrm{p}} \mathrm{u} \text {, a.e. in } \Omega \text {. }
$$

(b) $\left\langle\mathrm{L}_{\alpha, \mathrm{p}} \mathrm{u}, \phi v\right\rangle=2 \int_{\Omega} u \mathrm{~L}_{\alpha, \mathrm{p}} v$.

(c) For any $m \geqslant 0$ and $\xi \in \mathbb{R}$, define the truncated functions

$$
\mathcal{F}_{\mathfrak{m}}(\xi):=\max \{-\mathfrak{m}, \min \{m, \xi\}\}, \text { and } \mathcal{H}_{\mathfrak{m}}(\xi):=\xi-\mathcal{F}_{\mathfrak{m}}(\xi) .
$$

Then $\mathcal{F}_{\mathrm{m}}(\mathrm{u}), \mathcal{H}_{\mathrm{m}}(\mathrm{u}) \in \dot{\mathrm{W}}^{\alpha, p}(\Omega)$ and

$$
\left\|\mathcal{F}_{\mathfrak{m}}(u)\right\|_{\mathfrak{W}^{\alpha, p}(\Omega)}^{p} \leqslant\left\langle\mathrm{~L}_{\alpha, p} u, \mathcal{F}_{\mathfrak{m}}(u)\right\rangle \text {, and }\left\|\mathcal{H}_{\mathfrak{m}}(u)\right\|_{\mathfrak{W}^{\alpha, p}(\Omega)}^{p} \leqslant\left\langle\mathrm{~L}_{\alpha, p} u, \mathcal{H}_{m}(u)\right\rangle .
$$

The following fractional Morrey Sobolev inequality is essential to the proof of Theorem 1.2.

Lemma 2.4 ([20, Theorem 4.1]). Let $\alpha p>n$. Then there is a constant $C:=C(n, \alpha, p, \Omega)$ such that

$$
\|f\|_{L^{\infty}(\Omega)} \leqslant C\|f\|_{\dot{W}^{\alpha, p}(\Omega)}, \quad \forall f \in \dot{W}^{\alpha, p}(\Omega) .
$$

\section{Proofs of the main results}

In this section, we give the proofs of Theorem 1.1 and Theorem 1.2 by the so-called Moser's method. Following [1], we can also give the proofs with a stampacchia's type result. We omit here for their similarity.

\subsection{Proof of Theorem 1.1}

Let us begin with the proof of (a). There is no loss of generality in assuming that $|\Omega|=1$. We consider the following truncated function

$$
F_{\Psi}(\xi):= \begin{cases}|\xi|^{\gamma} & \text { as } 0 \leqslant|\xi|<\Psi, \\ \gamma \Psi \gamma-1(\xi-\Psi)+\Psi \gamma & \text { as } \xi \geqslant \Psi, \\ -\gamma \Psi \gamma^{\gamma-1}(\xi+\Psi)+\Psi \gamma & \text { as } \xi \leqslant-\Psi,\end{cases}
$$

where $\gamma \geqslant 1$ and $\Psi>0$ to be announced later. It is easy to check that $F_{\Psi}$ satisfies Lemma 2.3 (a), and hence $F_{\Psi}(u) \in \dot{W}^{\alpha, n / \alpha}(\Omega)$. It follows from Lemma 2.1, (2.1) and Lemma 2.3 that

$$
\begin{aligned}
\left\|F_{\Psi}(u)\right\|_{L^{q}(\Omega)}^{n / \alpha} & \leqslant C \int_{\Omega} \int_{\Omega} \frac{\left|F_{\Psi}(u)(y)-F_{\Psi}(u)(x)\right|^{n / \alpha}}{|y-x|^{2 n}} d y d x \\
& =C\left\langle L_{\alpha, n / \alpha} F_{\Psi}(u), F_{\Psi}(u)\right\rangle \\
& =2 C \int_{\Omega} F_{\Psi}(u)(x)\left[L_{\alpha, n / \alpha} F_{\Psi}(u)\right](x) d x \\
& \leqslant 2 C \int_{\Omega}\left|F_{\Psi}^{\prime}(u)(x)\right|^{n / \alpha-1} F_{\Psi}(u)(x) L_{\alpha, n / \alpha} u(x) d x \\
& =2 C \int_{\Omega}\left|F_{\Psi}^{\prime}(u)(x)\right|^{n / \alpha-1} F_{\Psi}(u)(x) f(x) d x .
\end{aligned}
$$


Since

$$
\mathrm{F}_{\Psi}(\mathrm{u}) \leqslant|u|^{\gamma} \text {, and }\left|\mathrm{F}_{\Psi}^{\prime}(\mathrm{u})\right| \leqslant \gamma|u|^{\mathrm{n} / \alpha-1} \text {, }
$$

the Hölder inequality gives

$$
\left\|F_{\Psi}(u)\right\|_{L^{q}(\Omega)}^{n / \alpha} \leqslant 2 C \gamma^{n / \alpha-1}\|g\|_{L^{s}(\Omega)}\left\||u|^{n / \alpha(\gamma-1)+1}\right\|_{L^{s^{\prime}}(\Omega)} .
$$

Hence

$$
\left\||u|^{\gamma}\right\|_{L^{q}(\Omega)}^{n / \alpha} \leqslant C \gamma^{n / \alpha-1}\|g\|_{L^{s}(\Omega)}\left\||u|^{\mathfrak{n} / \alpha(\gamma-1)+1}\right\|_{L^{s^{\prime}}(\Omega)},
$$

by taking $\Psi \rightarrow \infty$. Finally,

$$
\|u\|_{L^{\gamma q}(\Omega)} \leqslant C\left(\gamma^{n / \alpha-1}\|g\|_{L^{s}(\Omega)}\right)^{\frac{\alpha}{\gamma n}}\left(\int_{\Omega}|u(x)|^{(n / \alpha(\gamma-1)+1) s^{\prime}} d x\right) \frac{\alpha}{n \gamma s^{\prime}} .
$$

Applying Young's inequality to $p_{1}=\frac{n \gamma}{n(\gamma-1)+\alpha}$ and $p_{2}=\frac{n \gamma}{n-\alpha}$, (3.2) can be rewritten as

$$
\|u\|_{L^{\gamma q}(\Omega)} \leqslant C\left(\gamma^{n / \alpha-1}\|g\|_{L^{s}(\Omega)}\right)^{\frac{\alpha}{\gamma n}}\left(1+\int_{\Omega}|u(x)|^{n \gamma s^{\prime} / \alpha} d x\right) \frac{\alpha}{n \gamma \gamma s^{\prime}} .
$$

Denote by

$$
r_{k}=m^{k}:=\left(\frac{q \alpha}{n s^{\prime}}\right)^{k}, \quad I_{k}=\left(\int_{\Omega}|u|^{r_{k} q} d x\right)^{\frac{1}{r_{k} q}}, \quad \text { and } \quad J_{k}=\left(c r_{k}^{n / \alpha-1}\|f\|_{L^{s}(\Omega)}\right)^{\frac{\alpha}{r_{k}}} .
$$

It is obvious that $m>1$. We conclude from the fact $r_{k+1} \frac{n s^{\prime}}{\alpha}=r_{k} q$ and (3.3) that

$$
\mathrm{I}_{\mathrm{k}+1} \leqslant \mathrm{~J}_{\mathrm{k}+1}\left(1+\mathrm{I}_{\mathrm{k}}^{\mathrm{r}_{\mathrm{k}} \mathrm{q}}\right)^{\frac{1}{\mathrm{r}_{\mathrm{k}} \mathrm{q}}}
$$

Therefore, up to a re-normalization to obtain that $I_{0}=1$ and $I_{k} \geqslant 1$, one has

$$
\ln ^{I_{k+1}} \leqslant \ln ^{J_{k+1}}+\frac{1}{r_{k} q} \ln ^{\left(1+I_{k}^{r_{k} q}\right)} \leqslant \ln ^{J_{k+1}}+\frac{1}{r_{k} q}+\ln ^{I_{k}}
$$

Hence,

$$
\sum_{i=0}^{k+1}\left(\ln ^{\mathrm{I}_{i+1}}-\ln ^{\mathrm{I}_{i}}\right) \leqslant \sum_{i=0}^{k+1}\left(\ln ^{\mathrm{J}_{i+1}}+\frac{1}{r_{i} \mathrm{q}}\right)
$$

which implies that

$$
\ln ^{\mathrm{I}_{k+1}} \leqslant \sum_{i=1}^{k+1} \ln ^{\mathrm{J}_{i}}+\sum_{i=0}^{\mathrm{k}} \frac{1}{\mathrm{r}_{i} \mathrm{q}}+\ln ^{\mathrm{I}_{0}} \leqslant \sum_{i=1}^{\infty} \ln ^{\mathrm{J}_{i}}+\sum_{i=0}^{\infty} \frac{1}{\mathrm{r}_{i} \mathrm{q}}=\mathrm{N}<\infty .
$$

We have completed our proof after the following observation

$$
\mathrm{I}_{\mathrm{k}+1} \leqslant \mathrm{C}:=\mathrm{e}^{\mathrm{N}}<\infty \text {, and } \lim _{\mathrm{k} \rightarrow \infty} \mathrm{I}_{\mathrm{k}+1}=\|\mathrm{u}\|_{\mathrm{L}^{\infty}(\Omega)} .
$$

Next, we proceed the proof by showing (b). For any $\Psi>0$ and $\gamma>0$ will be fixed later, we define

$$
G_{\Psi}(\eta):= \begin{cases}e^{\gamma|\eta|}-1 & \text { as } 0 \leqslant|\eta|<\Psi, \\ \gamma e^{\gamma \Psi}(\eta-\Psi)+e^{\gamma \Psi}-1 & \text { as } \eta \geqslant \Psi \\ -\gamma e^{\gamma \Psi}(\eta+\Psi)+e^{\gamma \Psi}-1 & \text { as } \eta \leqslant-\Psi .\end{cases}
$$


It is easily seen that $\mathrm{G}_{\Psi}(\mathrm{u})$ satisfies Lemma 2.3 (a). A further use of Lemma 2.1 and Lemma 2.3, one has

$$
\begin{aligned}
& \left.\left\|G_{\Psi}(u)\right\|_{L^{q}(\Omega)}^{n / \alpha} \leqslant C<L_{\alpha, n / \alpha} G_{\Psi}(u), G_{\Psi}(u)\right\rangle \\
& =2 \mathrm{C} \int_{\Omega} \mathrm{G}_{\Psi}(\mathrm{u})(\mathrm{x})\left[\mathrm{L}_{\alpha, \mathrm{n} / \alpha} \mathrm{G}_{\Psi}(\mathrm{u})\right](\mathrm{x}) \mathrm{d} \mathrm{x} \\
& \leqslant 2 C \int_{\Omega}\left|G_{\Psi}^{\prime}(u)(x)\right|^{n / \alpha-1} G_{\Psi}(u)(x) L_{\alpha, n / \alpha} u(x) d x \\
& =2 \mathrm{C} \int_{\Omega_{1}^{c}}\left|G_{\psi}^{\prime}(u)(x)\right|^{n / \alpha-1} G_{\psi}(u)(x) g(x) d x \\
& +\int_{\Omega_{1}}\left|G_{\Psi}^{\prime}(u)(x)\right|^{n / \alpha-1} G_{\Psi}(u)(x) g(x) d x \\
& :=2 \mathrm{C}\left(\mathrm{K}_{1}+\mathrm{K}_{2}\right) \text {, }
\end{aligned}
$$

where $\Omega_{1}:=\{x \in \Omega:|u(x)| \geqslant \Psi\}$.

Set

$$
\Omega_{11}^{c}:=\left\{x \in \Omega_{1}^{c}: G_{\Psi}(u)(x) \geqslant 1\right\}, \text { and } \Omega_{12}^{c}:=\left\{x \in \Omega_{1}^{c}: G_{\Psi}(u)(x)<1\right\} .
$$

We see at once that

$$
\left(\mathrm{G}_{\Psi}(\mathrm{u})(\mathrm{x})+1\right)^{\mathrm{n} / \alpha-1} \leqslant 2^{\mathrm{n} / \alpha-1}\left(\left(\mathrm{G}_{\Psi}(\mathrm{u})(\mathrm{x})\right)^{\mathrm{n} / \alpha-1}+1\right) \text { as } \mathrm{x} \in \Omega_{11}^{\mathrm{c}},
$$

and

$$
\left(G_{\Psi}(u)(x)+1\right)^{n / \alpha-1} \leqslant 2^{n / \alpha-1} \leqslant 2^{n / \alpha-1}\left(\left(G_{\Psi}(u)(x)\right)^{n / \alpha-1}+1\right) \text { as } x \in \Omega_{12}^{c} .
$$

Since

$$
\left|G_{\Psi}^{\prime}(u)(x)\right|=\gamma\left(G_{\Psi}(u)(x)+1\right) \text { as } x \in \Omega_{1}^{c},
$$

the Hölder inequality shows that

$$
\begin{aligned}
\mathrm{K}_{1} & \leqslant 2^{\mathrm{n} / \alpha-1} \gamma^{\mathrm{n} / \alpha-1} \int_{\Omega_{1}^{\mathrm{c}}}\left[\left(\mathrm{G}_{\Psi}(\mathrm{u})(x)\right)^{\mathrm{n} / \alpha} \mathrm{g}(\mathrm{x})+\left(\mathrm{G}_{\Psi}(\mathrm{u})(x)\right) g(x)\right] \mathrm{d} x \\
& \leqslant(2 \gamma)^{\mathrm{n} / \alpha-1}\|\mathrm{~g}\|_{L^{\mathrm{s}}(\Omega)}\left\|\mathrm{G}_{\Psi}(\mathrm{u})\right\|_{\mathrm{L}^{\mathrm{q}}(\Omega)}^{\mathrm{n} / \alpha}+(2 \gamma)^{\mathrm{n} / \alpha-1} \int_{\Omega} \mathrm{G}_{\Psi}(\mathrm{u})(x) g(x) \mathrm{d} x \\
& :=\mathrm{K}_{11}+\mathrm{K}_{12}
\end{aligned}
$$

and hence

$$
\mathrm{K}_{12} \leqslant(2 \gamma)^{\mathrm{n} / \alpha-1}\|g\|_{\mathrm{L}^{s}(\Omega)}\left\|\mathrm{G}_{\Psi}(\mathrm{u})\right\|_{\mathrm{L}^{s^{\prime}}(\Omega)} \leqslant(2 \gamma)^{\mathrm{n} / \alpha-1}\|g\|_{\mathrm{L}^{\mathrm{s}}(\Omega)}\left\|\mathrm{G}_{\Psi}(\mathrm{u})\right\|_{\mathrm{L}^{\mathrm{q}}(\Omega)}|\Omega|^{\frac{\mathrm{n}-\alpha}{\alpha q}} .
$$

By the Minkowski inequality, $\mathrm{K}_{12}$ can be further estimated as

$$
K_{12} \leqslant(2 \gamma)^{n / \alpha-1}\left(\frac{\alpha}{n}\|g\|_{L^{s}(\Omega)}\left\|G_{\Psi}(u)\right\|_{L^{q}(\Omega)}^{n / \alpha}|\Omega|^{\frac{n(n-\alpha)}{\alpha^{2} q}}+\frac{n-\alpha}{n}\|g\|_{L^{s}(\Omega)}\right) .
$$

Therefore,

$$
\begin{aligned}
& \mathrm{K}_{1} \leqslant 2^{\mathrm{n} / \alpha} \gamma^{\mathrm{n} / \alpha-1}\|g\|_{\mathrm{L}^{\mathrm{s}}(\Omega)}\left\|\mathrm{G}_{\Psi}(\mathrm{u})\right\|_{\mathrm{L}^{\mathrm{q}}(\Omega)}^{\mathrm{n} / \alpha}+\frac{\alpha}{\mathrm{n}}\|g\|_{\mathrm{L}^{\mathrm{s}}(\Omega)}\left\|\mathrm{G}_{\Psi}(\mathrm{u})\right\|_{\mathrm{L}^{\mathrm{q}}(\Omega)}^{\mathrm{n} / \alpha}|\Omega|^{\frac{\mathrm{n}(\mathrm{n}-\alpha)}{\alpha^{2} \mathrm{q}}} \\
& +\frac{n-\alpha}{n}\|g\|_{L^{s}(\Omega)} \\
& \leqslant 2^{n / \alpha} \gamma^{n / \alpha-1}\|g\|_{L^{s}(\Omega)}\left\|G_{\Psi}(u)\right\|_{L^{q}(\Omega)}^{n / \alpha}\left(1+\frac{\alpha}{n}|\Omega|^{\frac{n(n-\alpha)}{\alpha^{2} q}}\right) \\
& +2^{n / \alpha} \gamma^{n / \alpha-1} \frac{n-\alpha}{n}\|g\|_{L^{s}(\Omega)} .
\end{aligned}
$$

For the term $\mathrm{K}_{2}$, we first note that

$$
\min _{\Omega_{1}} G_{\Psi}(u)=G_{\Psi}(\Psi)=G_{\Psi}(-\Psi)=e^{\gamma \Psi}-1 .
$$


Choosing $\gamma \Psi>1$, Hölder's inequality gives

$$
\begin{aligned}
K_{2} & \leqslant \frac{\left(\gamma e^{\gamma \Psi}\right)^{n / \alpha-1}}{G_{\Psi}(\Psi)^{n / \alpha-1}} \int_{\Omega_{1}} G_{\Psi}(u(x))^{n / \alpha} g(x) d x \\
& \leqslant \gamma^{n / \alpha-1}\left(\frac{e^{\gamma \Psi}}{e^{\gamma \Psi}-1}\right)^{n / \alpha-1}\|g\|_{L^{s}(\Omega)}\left\|G_{\Psi}(u)\right\|_{L^{q}(\Omega)}^{n / \alpha} \\
& \leqslant(2 \gamma)^{n / \alpha-1}\|g\|_{L^{s}(\Omega)}\left\|G_{\Psi}(u)\right\|_{L^{q}(\Omega)}^{n / \alpha} .
\end{aligned}
$$

On account of the above estimates, we have

$$
\begin{aligned}
\left\|G_{\Psi}(u)\right\|_{L^{q}(\Omega)}^{n / \alpha} \leqslant & 2^{n / \alpha}(\gamma)^{n / \alpha-1}\|g\|_{L^{s}(\Omega)}\left\|G_{\Psi}(u)\right\|_{L^{q}(\Omega)}^{n / \alpha}\left(2+\frac{\alpha}{n}|\Omega|^{\frac{n(n-\alpha)}{\alpha^{2} q}}\right) \\
& +\frac{n-\alpha}{n} 2^{n / \alpha}(\gamma)^{n / \alpha-1}\|g\|_{L^{s}(\Omega)} .
\end{aligned}
$$

Therefore,

$$
\left\|\mathrm{G}_{\Psi}(\mathrm{u})\right\|_{\mathrm{L}^{\mathrm{q}}(\Omega)}^{\mathrm{n} / \alpha} \leqslant \mathrm{C}^{\frac{\mathrm{n}}{\alpha}}\left(\mathrm{n}, \alpha, \mathrm{q},\|\mathrm{g}\|_{\mathrm{L}^{\mathrm{s}}(\Omega)}, \Omega\right),
$$

by choosing $\gamma$ small enough. This forces

$$
\left\|\mathrm{G}_{\Psi}(\mathrm{u})\right\|_{\mathrm{Lq}^{\mathrm{q}}(\Omega)}=\left\|e^{\gamma|\mathfrak{u}|}-1\right\|_{\mathrm{Lq}^{\mathrm{q}}(\Omega)} \leqslant \mathrm{C}<\infty,
$$

after taking $\Psi \rightarrow \infty$. Thus, we get our desired result by taking $\beta=\gamma q>0$.

Finally, we give the proof of (c). For $\Psi>0$ big enough to be determined later, we consider the function (3.1) with $\gamma=\frac{s^{*}}{q}$. We deduce from the fact $\frac{q}{q-1} \leqslant s<\frac{\alpha q}{\alpha q-n}$ that $\gamma \geqslant 1$. By the similar analysis as that in the proof of (a), we have

$$
\left\|F_{\Psi}(u)\right\|_{L^{q}(\Omega)}^{n / \alpha} \leqslant 2 C \gamma^{\frac{n}{\alpha}-1} \mid g \|_{L^{s}(\Omega)}\left(\int_{\Omega}|u(x)|^{s^{\prime}\left((\gamma-1) \frac{n}{\alpha}+1\right)} d x\right)^{\frac{1}{s^{\prime}}} .
$$

Hence,

$$
\left(\int_{\Omega}|u(x)|^{\gamma q} d x\right)^{\frac{n}{q-\alpha}} \leqslant 2 C \gamma^{\frac{n}{\alpha}-1}\|g\|_{L^{s}(\Omega)}\left(\int_{\Omega}|u(x)|^{s^{\prime}\left((\gamma-1) \frac{n}{\alpha}+1\right)} d x\right)^{\frac{1}{s^{\prime}}},
$$

by taking $\Psi \rightarrow \infty$. It follows from the fact

$$
\gamma q=s^{\prime}\left((\gamma-1) \frac{n}{\alpha}+1\right)=s^{*}, \text { and } \frac{n}{\alpha q}-\frac{1}{s^{\prime}}=\frac{\alpha q-s(\alpha q-n)}{\alpha q s}>0,
$$

that

which gives

$$
\|u\|_{L_{\mathrm{s}^{*}(\Omega)}^{\frac{n-\alpha}{\alpha}}}^{\frac{n}{\alpha}} \leqslant 2 C \gamma^{\mathrm{n} / \alpha-1}\|g\|_{L^{s}(\Omega)}
$$

$$
\|u\|_{L^{s^{*}}(\Omega)} \leqslant C_{3}\|g\|_{L^{s}(\Omega)}^{\frac{\alpha}{n-\alpha}}
$$

\subsection{Proof of Theorem 1.2}

Using the similar arguments as that of Section 3.1, we can prove Theorem 1.2. In fact, for the function defined in (3.1) with $\gamma=1$ and $\Psi>0$, by Lemma 2.3 and Lemma 2.4, we have

$$
\begin{aligned}
\left\|F_{\Psi}(u)\right\|_{L^{\infty}(\Omega)} & \leqslant 2 \tilde{C}\left(\int_{\Omega}\left|F_{\Psi}^{\prime}(u(x))\right|^{p-1} F_{\Psi}(u(x)) g(x) d x\right)^{1 / p} \\
& \leqslant 2 \tilde{C}\|u\|_{L^{\infty}(\Omega)}^{1 / p}\|g\|_{L^{1}(\Omega)}^{1 / p} .
\end{aligned}
$$

Taking $\Psi \rightarrow \infty$, one has

$$
\|u\|_{L^{\infty}(\Omega)} \leqslant 2 \tilde{C}\|u\|_{L^{\infty}(\Omega)}^{1 / p}\|g\|_{L^{1}(\Omega)}^{1 / p} .
$$


Hence

$$
\|u\|_{L^{\infty}(\Omega)} \leqslant 2 \tilde{C}\|g\|_{L^{1}(\Omega)}^{p^{\prime} / p} .
$$

Theorem 1.2 has been proved by taking $C=2 \tilde{C}\|g\|_{L^{1}(\Omega)}^{p^{\prime} / p}$.

\section{Acknowledgment}

This work was partially supported by the key Laboratory of Complex Systems and Intelligent Computing in University of Shandong (Linyi University), the Applied Mathematics Enhancement Program of Linyi University and the National Natural Science Foundation of China (11771195).

\section{References}

[1] B. Barrios, I. Peral, S. Vita, Some remarks about the summability of nonlocal nonlinear problems, Adv. Nonlinear Anal., 4 (2015), 91-107. 1, 1, 2, 2, 3

[2] C. Bjorland, L. Caffarelli, A. Figalli, Nonlocal tug-of-war and the infinity fractional Laplacian, Comm. Pure Appl. Math., 65 (2012), 337-380. 1

[3] K. Bogdan, K. Burdzy, Z.-Q. Chen, Censored stable processes, Probab. Theory Related Fields, 127 (2003), 89-152. 1

[4] L. A. Caffarelli, S. Salsa, L. Silvestre, Regularity estimates for the solution and the free boundary of the obstacle problem for the fractional Laplacian, Invent. Math., 171 (2008), 425-461. 1

[5] A. Di Castro, T. Kuusi, G. Palatucci, Nonlocal Harnack inequalities, J. Funct. Anal., 267 (2014), 1807-1836. 1

[6] E. Di Nezza, G. Palatucci, E. Valdinoci, Hitchhiker's guide to the fractional Sobolev spaces, Bull. Sci. Math., 136 (2012), 521-573. 2, 2

[7] A. Garroni, R. V. Kohn, Some three-dimensional problems related to dielectric breakdown and polycrystal plasticity, R. Soc. Lond. Proc. Ser. A Math. Phys. Eng. Sci., 459 (2003), 2613-2625. 2

[8] Q.-Y. Guan, Z.-M. Ma, Reflected symmetric $\alpha$-stable processes and regional fractional Laplacian, Probab. Theory Related Fields, 134 (2006), 649-694. 1

[9] R. Hurri-Syrjänen, A. V. Vähäkangas, Fractional Sobolev-Poincar and fractional Hardy inequalities in unbounded John domains, Mathematika, 61 (2015), 385-401. 2

[10] A. Iannizzotto, S.-B. Liu, K. Perera, M. Squassina, Existence results for fractional p-Laplacian problems via Morse theory, Adv. Calc. Var., 9 (2016), 101-125. 1

[11] N. S. Landkof, Foundations of modern potential theory, Translated from the Russian by A. P. Doohovskoy, Die Grundlehren der mathematischen Wissenschaften, Band 180. Springer-Verlag, New York-Heidelberg, (1972). 1

[12] T. Leonori, I. Peral, A. Primo, F. Soria, Basic estimates for solution of elliptic and parabolic equations for a class of nonlocal operators, Discrete Contin. Dyn. Syst. Ser. A, 35 (2015), 6031-6068. 1, 1

[13] E. Lindgren, P. Lindqvist, Fractional eigenvalues, Calc. Var. Partial Differential Equations, 49 (2014), 795-826. 1

[14] X. Ros-Oton, J. Serra, The Dirichlet problem for the fractional Laplacian: regularity up to the boundary, J. Math. Pures Appl., 101 (2014), 275-302. 1

[15] S.-G. Shi, J. Xiao, On fractional capacities relative to bounded open Lipschitz sets, Potential Anal., 45 (2016), 261-298. 1

[16] S.-G. Shi, J. Xiao, Fractional capacities relative to bounded open Lipschitz sets complemented, Calc. Var. Partial Differential Equations, 56 (2017), 22 pages. 1

[17] P. Shvartsman, On Sobolev extension domains in $\mathbb{R}^{n}$, J. Funct. Anal., 258 (2010), 2205-2245. 2

[18] E. M. Stein, Singular integrals and differentiability properties of functions, Princeton Mathematical Series, No. 30 Princeton University Press, Princeton, N.J., (1970). 1

[19] H. Triebel, Function spaces and wavelets on domains, EMS Tracts in Mathematics, European Mathematical Society (EMS), Zürich, (2008). 2

[20] J. Xiao, Z. Zhai, Fractional Sobolev, Moser-Trudinger Morrey-Sobolev inequalities under Lorentz norms, Problems in mathematical analysis, J. Math. Sci. (N.Y.), 166 (2010), 357-376. 2.4

[21] Y. Zhou, Fractional Sobolev extension and imbedding, Trans. Amer. Math. Soc., 367 (2015), 959-979. 2 